\title{
Research Progress of Circulating Tumor Cells in the Evaluation of Early Cholangiocarcinoma
}

\author{
Quan Zheng1,2, Ying Xiong1*+, Jinku Zhang2*† \\ ${ }^{1}$ Department of Gastroenterology, Baoding First Central Hospital, Baoding 071000, China \\ ${ }^{2}$ Hebei Key Laboratory of Molecular Pathology and Early Diagnosis of Tumor, Baoding, China \\ ${ }^{\dagger}$ These authors contributed equally to this work
}

*Corresponding author: Ying Xiong, xy_spring@163.com; Jinku Zhang, zjkblk@sina.com

\begin{abstract}
Epidemiological data show that the incidence rate of cholangiocarcinoma (CCA) has been increasing over the past 20 years. Due to its concealment of clinical manifestations, most patients are diagnosed with CCA at a late stage. On this premise, this review not only discusses the research progress related to CCA diagnosis, but also focuses on the concept of circulating tumor cell (CTC), its evaluation value, application, and prospects for the early diagnosis of extrahepatic cholangiocarcinoma.
\end{abstract}

Keywords: Circulating tumor cell; Biliary tract; Cholangiocarcinoma; Diagnosis

Publication date: September 2021; Online publication: September 30, 2021

\section{Introduction}

Among the most common primary malignant hepatobiliary malignancies, cholangiocarcinoma (CCA) ranks second. There are strong epidemiological data showing that the incidence rate has increased steadily over the past 20 years ${ }^{[1]}$. CCA is generally divided into intrahepatic, perihilar, and distal cholangiocarcinoma according to its anatomical location along the biliary tree. Histological, clinical, and molecular data have suggested that there may be genetic differences in the biological behavior among these CCA subtypes, other than their different anatomical locations. In addition, due to their anatomical location, growth pattern, and lack of clear diagnostic criteria, their clinical processes and invasive behaviors are very different along with a difficulty in diagnosing ${ }^{[2,3]}$.

The incidence rate of intrahepatic cholangiocarcinoma has been increasing in the recent 20 years, but the incidence rate of extrahepatic bile duct cancer around the hilar has been stable ${ }^{[2,3]}$. The main reasons for the steady increase in the incidence rate of CCA is first, because of its silent clinical features, early diagnosis is difficult, treatment methods are limited, and the prognosis is poor. The median survival time is less than 24 months. Second, surgery is the only possible curative treatment, but it is only applicable in the early stage of the disease. Third, there is no reliable staging system to achieve optimal treatment allocation and prediction ${ }^{[2,3]}$.

Most CCA patients have symptoms only in the late stage of the disease, and their clinical manifestations depend on the tumor stage, tumor location, and growth pattern. The diagnosis of CCA requires a high degree of suspicion in appropriate clinical settings as well as a confirmatory combination of clinical, laboratory, endoscopic, and radiological data. CCA is difficult to diagnose because of its "silent" clinical features, the low specificity of most diagnostic methods, and the lack of an absolute diagnostic criteria ${ }^{[1,2]}$. Therefore, the vast majority of patients are in advanced stage of cancer when diagnosed with CCA, and the 
survival benefits of palliative treatment are very limited. Therefore, this review aims to focus more on how to improve the rate of early diagnosis of CCA in order not to miss the golden time for diagnosis and treatment.

\section{Progress}

Nowadays, the medical community has made progress in the diagnosis of cholangiocarcinoma. In terms of molecular prognosis prediction, a recent study showed that the nuclear expression of S100A4 in tumor ducts is associated with adverse reactions from surgical treatment in CCA patients ${ }^{[4]}$. In terms of circulating markers, the diagnostic role of Cyfra 21-1 (a fragment of cytokeratin 19) is considered to be related to circulating tumor cells in biliary carcinoma ${ }^{[5]}$. In terms of early diagnosis, recent studies have used deep sequencing technology to identify candidate carcinogenic drivers of intrahepatic CCA, and gene fusion involving FGFR24 has been proven to be able to induce tumor formation in the experimental model of intrahepatic $\mathrm{CCA}^{[6]}$. Other common genetic defects include IDH and KRAS mutations.

\section{Circulating tumor cell (CTC)}

\subsection{Definition and separation method of CTC}

As a potential new biomarker, CTC has been widely used for many solid tumors. CTCs are a type of tumor cell that are released into the peripheral blood circulation from solid tumors spontaneously or due to diagnosis and treatment. They have the characteristics of tumor initiation, especially when they survive in the peripheral blood and aggregate to form small tumor thrombi; they are more likely to promote metastases, leading to cancer recurrence ${ }^{[7]}$. Moreover, the higher the number of CTCs in peripheral blood, the higher the possibility of cancer metastases or the higher the degree of deterioration. Therefore, the presence and quantity of CTCs in peripheral blood represent not only the ability of a primary tumor to infiltrate into blood vessels, but also the possibility of metastases. At present, many studies have evaluated the role and significance of CTCs as biomarkers in predicting recurrence, prognosis evaluation, and treatment response of different tumors. Most of them use CTC count as a surrogate indicator of metastatic potential and believe that the identification and quantification of CTC alone can effectively predict the prognosis of cancer. Therefore, CTC count is considered as a marker of poor prognosis ${ }^{[1,8]}$.

Epithelial cell adhesion molecule (EpCAM) is a type of transmembrane glycoprotein that is expressed only in cancers of epithelial origin, but not in normal cells from the circulatory system. According to the specificity of antigen and antibody, most CTC isolation methods rely on it for CTC capture while other enrichment methods depend on the physical properties of cells (such as cell size, etc.). At the same time, there are increasing studies to replace EpCAM for CTC separation as exemplified in a new study that attempted to evaluate the efficacy of EpCAM in 65 patients with advanced HCC or metastatic cholangiocarcinoma (CCA) and the feasibility of using a new glycosaminoglycan, SCH45, as a probe to isolate CTCs from peripheral blood of patients ${ }^{[9]}$.

\subsection{Application of CTC in diagnosing cancer}

In 2016, there was a study that has made great progress in using circulating tumor cells and cell free DNA to monitor the evolution of gastrointestinal cancer, especially colorectal cancer ${ }^{[10]}$. In this study, liquid biopsy technology was introduced to capture and test CTC. It was found that CTCs have important clinical significance in early diagnosis and efficacy monitoring. In view of that, Li and other researchers prospectively evaluated 136 patients with gastric cancer ${ }^{[11]}$. After 6 weeks of chemotherapy, the level of CTC $(\geqslant 3 / 7.5 \mathrm{ml})$ was closely related to the objective response rate $(\mathrm{P}=0.016)$ and disease control rate $(\mathrm{P}$ $=0.013)$. In addition, it could independently predict the short progression free survival and overall survival. 
It is worth mentioning that the prognosis of patients with lower CTC levels after treatment is better, but significantly worse for patients with higher CTC levels. The study concluded that the level of CTC after treatment is helpful to evaluate the curative effect and predict the prognosis of patients with gastric cancer; in addition, a high CTC level during treatment may be associated with poor prognosis. Other than that, the changes in CTCs after treatment may assist in the quick identification of ineffective treatment and poor prognosis.

When the location of primary tumor or metastatic tumor is unknown, it is extremely important to identify the tissue of origin to determine the anatomical location of the primary tumor, micrometastases, and metastases to guide medical imaging and tissue examination ${ }^{[12]}$. Some studies have analyzed the genomic map of CTC in order to determine the organ location in metastases ${ }^{[13]}$. Several genomic signatures are associated with the specific site of metastases ${ }^{[14,15]}$. Therefore, expression signatures seem to be more likely to provide information from specific parts of CTCs. Tumor cells that are hosted in specific sites may manifest specific expression patterns, which is caused by the crosstalk of microenvironment ${ }^{[16]}$. The identification of CTC components in patients with metastatic breast cancer has confirmed this hypothesis and emphasized that Notch pathway, immunomodulatory pathway (tumor necrosis factor, interleukin- $1 \beta$, and nuclear factor kappa B), inflammatory pathway (chemokine CXCL8, chemokine receptor CXCR4, and CD86), and mitogenic pathway (platelet-derived growth factor BB) are the marker pathways of CTCs associated with brain metastases ${ }^{[17]}$. In a study of breast cancer cases with skeletal and visceral organ progression, the androgen receptor expression in CTC was higher than that in the control group (AR). The activation of signal is related to breast cancer, which mainly metastasizes to bones, undoubtedly revealing a new way of diagnosis and treatment ${ }^{[18]}$.

Ten years ago, the term "liquid biopsy" was first introduced to describe the effect of circulating tumor cells in the blood of cancer patients. However, at present, the technology has expanded to analyzing circulating tumor DNA (CtDNA), detection of microRNA, microbubbles, and even platelets. The detection of microRNA, microbubbles, and even platelets all contain tumor derived information, thus making liquid biopsy technology have important clinical significance in cancer diagnosis, prognosis, prediction of curable diseases, efficacy monitoring, and so on ${ }^{[19-22]}$.

\subsection{Diagnostic efficacy of CTC for CCA}

There are several data on the prognostic role of CTC in CCA. In Hepatology, Yang and other researchers examined the relationship among the number of CTCs, more aggressive tumor features, and lower survival rate in CCA patients ${ }^{[23]}$. Although that was not the first time that CTC in CCA has been analyzed, it is the largest analysis of this kind so far, especially the inclusion of correlation with clinical results in that study. The study used Janssen diagnostics, the first FDA approved CTC counting system, to count the CTCs in 88 patients. However, the study had some limitations. The first limitation is the capture method; as it depends on the expression of EpCAM, it is impossible to count the non-EpCAM expression of CTC, thus unable to detect the corresponding cancer cells or the actual number of CTCs would be underestimated. Paired analysis of EpCAM expression and CTC count in tumor tissues can help us understand the extent of this restriction. The second is the patients that were included in the study and the different treatment methods of the patients; patients receiving early surgical treatment to those with metastatic diseases receiving chemotherapy were included. Although CTC, as an independent biomarker, is not necessarily related to the cancer stage and treatment method, the different prognosis of the patients would still affect the quantitative level of CTC. In addition, some of the proposed subanalyses might not have accurately captured prognostic differences. Nevertheless, this study provides preliminary evidence for the prognostic role of CTC in CCA, and further suggests that tumor biomarkers in the circulatory system may play an important role in the decision-making of patients with liver cancer. 
As the number of CTC is minimal in the blood circulatory system, it is more likely to be detected in patients with metastatic diseases, thus endearing it to have certain limitations as a biomarker for the early diagnosis of cancer at an early stage. In addition, there is a lack of large sample clinical research to explore and demonstrate. If the sensitivity of CTC can be significantly improved by larger blood collection or more advanced detection technology, CTC may become an effective tool for the early detection of cancer. This may be more reliable than cfDNA because it is not hindered by DNA mutations associated with aging and it can represent the DNA "signal" released by intact living tumor cells rather than dying ones.

In January 2021, the latest achievements of Ahn JC and others, who studied the significance of CTC as a biomarker for diagnosis, prognosis, and efficacy monitoring of CCA ${ }^{[24]}$, pointed out that currently available tests such as alpha fetoprotein have limited accuracy as biomarkers for the diagnosis or prognosis of liver cancer. In addition to that, liver biopsy provides tissue that can reveal tumor biology, but it is not a conventional diagnostic method because of its invasiveness and the risk of tumor implantation, especially in early-stage patients. Moreover, due to the heterogeneity within the tumor, liver biopsy has limitation in revealing the overall tumor biology. The article focuses on liquid biopsy, which is an effective method for early detection and diagnosis of liver cancer. CTC is a type of circulating cancer cell from primary tumors or metastases. It can be detected by simple peripheral blood and may show the global characteristics of tumor characteristics. Liquid biopsy has great potential in the early diagnosis and treatment of liver cancer patients.

\section{Prospect}

A series of studies on the relationship between CTC and CCA has shown clear logic. CTC may become an effective tool in the clinical application for CCA patients. The role of CTC count is not only limited to evaluating the prognosis of patients with CCA, but also has good diagnostic value in differentiating benign from malignant biliary tract tumors prior to surgery. It can be used as an auxiliary reference for traditional tumor markers.

With intuitive and efficient examinations of the extrahepatic bile duct mucosa by choledochoscopy, there are more opportunities to explore the correlation between circulating tumor cells and early extrahepatic cholangiocarcinoma. In cases where CTC is found in high-risk groups having extrahepatic cholangiocarcinoma (bile duct cyst and stone, liver cirrhosis, hepatitis B, hepatitis $\mathrm{C}$ virus, and smoking), choledochoscopy can be used to determine the cause of the disease so as to improve the detection rate of early extrahepatic cholangiocarcinoma.

With the continuous research progress of CTC, the level of CTC in the diagnosis of early extrahepatic cholangiocarcinoma may also have a breakthrough, in which its potential application and prospects are very considerable.

\section{Funding}

This work was supported by Baoding Technology Program (project number: 2041ZF084).

\section{Disclosure statement}

The authors declare that there is no conflict of interest.

\section{References}

[1] Roberto G, Augusto V, 2016, Circulating Tumor Cells and Cholangiocarcinoma. Hepatology, 63: 23 25. 
[2] Blechacz B, Komuta M, Roskams T, et al., 2011, Clinical Diagnosis and Staging of Cholangiocarcinoma. Nat Rev Gastroenterol Hepatol, 8: 512-522.

[3] Banales Jesus M, Marin Jose JG, Lamarca A, et al., 2020, Cholangiocarcinoma 2020: The Next Horizon in Mechanisms and Management. Nat Rev Gastroenterol Hepatol, 17: 557-588.

[4] Fabris L, Cadamuro M, Moserle L, et al., Nuclear Expression of S100A4 Calcium-Binding Protein Increases Cholangiocarcinoma Invasiveness and Metastasization. Hepatology, 54: 890-899.

[5] Chapman MH, Sandanayake NS, Andreola F, et al., 2011, Circulating CYFRA 21-1 is a Specific Diagnostic and Prognostic Biomarker in Biliary Tract Cancer. J Clin Exp Hepatol, 1: 6-12.

[6] Sia D, Losic B, Moeini A, et al., 2015, Massive Parallel Sequencing Uncovers Actionable FGFR2PPHLN1 Fusion and ARAF Mutations in Intrahepatic Cholangiocarcinoma. Nat Commun, 6: 6087.

[7] Aceto N, Bardia A, Miyamoto DT, et al., 2014, Circulating Tumor Cell Clusters are Oligoclonal Precursors of Breast Cancer Metastasis. Cell, 158: 1110-1122.

[8] Labgaa I, Villanueva A, 2015, Liquid Biopsy in Liver Cancer. Discov Med, 19: 263-273.

[9] Gopinathan P, Chiang NJ, Bandaru A, et al., 2020, Exploring Circulating Tumor Cells in Cholangiocarcinoma Using a Novel Glycosaminoglycan Probe on a Microfluidic Platform. Adv Healthc Mater, 9: e1901875.

[10] Pantel K, Alix-Panabières C, 2017, Liquid Biopsy in 2016: Circulating Tumour Cells and Cell-Free DNA in Gastrointestinal Cancer. Nat Rev Gastroenterol Hepatol, 14: 73-74.

[11] Li Y, Gong J, Zhang Q, et al., 2016, Dynamic Monitoring of Circulating Tumour Cells to Evaluate Therapeutic Efficacy in Advanced Gastric Cancer. Br J Cancer, 114: 138-145.

[12] Keller L, Pantel K, 2019, Unravelling Tumour Heterogeneity by Single-Cell Profiling of Circulating Tumour Cells. Nat Rev Cancer, 19: 553-567.

[13] Gulbahce, N et al. Quantitative whole genome sequencing of circulating tumor cells enables personalized combination therapy of metastatic cancer[J]. Cancer Res. 77, 4530-4541 (2017).

[14] Wrage M, Ruosaari S, Eijk PP, et al., 2009, Genomic Profiles Associated with Early Micrometastasis in Lung Cancer: Relevance of 4q Deletion. Clin Cancer Res, 15: 1566-1574.

[15] Wikman H, Lamszus K, Detels N, et al., 2012, Relevance of PTEN Loss in Brain Metastasis Formation in Breast Cancer Patients. Breast Cancer Res, 14: R49.

[16] Fluegen G, Avivar-Valderas A, Wang Y, et al., 2017, Phenotypic Heterogeneity of Disseminated Tumour Cells is Preset by Primary Tumour Hypoxic Microenvironments. Nat Cell Biol, 19: 120-132.

[17] Boral D, Vishnoi M, Liu HN, et al., 2017, Molecular Characterization of Breast Cancer CTCs Associated with Brain Metastasis. Nat Commun, 8: 196.

[18] Aceto N, Bardia A, Wittner BS, et al., 2018, AR Expression in Breast Cancer CTCs Associates with Bone Metastases. Mol Cancer Res, 16: 720-727.

[19] Chang YS, di Tomaso E, McDonald DM, et al., 2000, Mosaic Blood Vessels in Tumors: Frequency of Cancer Cells in Contact with Flowing Blood. Proc Natl Acad Sci, 97: 14608-14613.

[20] Lucci A, Hall CS, Lodhi AK, et al., 2012, Circulating Tumour Cells in Non-Metastatic Breast Cancer: A Prospective Study. Lancet Oncol, 13: 688-95.

[21] Rack B, Schindlbeck C, Jückstock J, et al., 2014, Circulating Tumor Cells Predict Survival in Early Average-to-High Risk Breast Cancer Patients. J Natl Cancer Inst, 106: undefined.

[22] Cohen SJ, Punt CJA, Iannotti N, et al., 2008, Relationship of Circulating Tumor Cells to Tumor Response, Progression-Free Survival, and Overall Survival in Patients with Metastatic Colorectal 
Cancer. J Clin Oncol, 26: 3213-3221.

[23] Yang JD, Campion MB, Liu MC, et al., 2016, Circulating Tumor Cells are Associated with Poor Overall Survival in Patients with Cholangiocarcinoma. Hepatology, 63: 148-58.

[24] Ahn JC, Teng PC, Chen PJ, et al., 2021, Detection of Circulating Tumor Cells and Their Implications as a Biomarker for Diagnosis, Prognostication, and Therapeutic Monitoring in Hepatocellular Carcinoma. Hepatology, 73: 422-436. 